\title{
The Research of Mobile Teaching Platform Based on Intelligent Mobile Phone
}

\author{
LI Yuefeng ${ }^{1, \text { a }}$ \\ ${ }^{1}$ GLOBAL INSTITUTE OF SOFTWARE TECHNOLOGY, Suzhou 215163,China
}

aliyuefeng@126.com

Keywords: Smart phones; Mobile teaching; Android; Mobile Learning

\begin{abstract}
Along with the level of Mobile terminals and network communication is increasingly common, Mobile Learning (Mobile Learning) gradually become E - Learning research and application of the latest development trend and one of the hot research topic. The development and use of mobile learning platform for teachers and students in colleges and universities to provide a kind of brand-new teaching mode, implement the reform of the traditional teaching mode, has a very broad application prospects. But to apply mobile learning system in the teaching of the colleges and universities, and make it become an efficient way of learning, to the whole of it conduct the thorough research to the learning system, teaching mode. This article through to analysis the main problems existing in the college classroom teaching research, comprehensive considering the popularity of mobile phone users and mobile phone terminal learning broad, put forward a learning system based on mobile platform to realize the mobile teaching experimental research.
\end{abstract}

\section{Introduction}

Along with the development of informatization of education technology, mobile learning theory in the academia and practice has received widespread attention, and with the popularity of smart mobile terminals and the time away from the computer more and more teachers and students present situation, the existing learning system can't satisfy the needs of teachers and students learning anytime and anywhere, and mobile learning is the teachers and students use mobile phone, PDA, car terminal, mobile terminal equipment such as notebook computers via SMS, WAP, VPN, GPRS, 3G and WIFI to connect a variety of communication methods and teaching system, which will become a safe campus Intranet expand wan, achieve learning anytime and anywhere [1-2]. As a result, mobile learning as a new form of learning arises at the historic moment.

In this paper, using the current relatively popular in the Android technology, with the aid of Linux system, the characteristics of this group of students, and school problems in the teaching process is analyzed, for college students to set up a learning system based on mobile platform. In system design stage, first has carried on the overall design of the whole system, then analyze the teaching system of business process, found in the redundancy in the process of business operations, on the process of restructuring. The purpose of this study is to discuss the application of mobile platform in the teaching to achieve the best teaching effect, which can let teachers and students to achieve a new way of communication. The law application system for instrument design and implementation of a new way of communication.

\section{Android mobile development technology}

Android because of its open source and powerful features did a lot of attention [3]. On formal listed a few short years, widespread Android operating system, become the world's most popular mobile operating system. Now, the wide application of Android offers the convenience of mobile Internet means not only can be used on a smartphone, can also be used for the mobile Internet terminals, and other handheld devices [4]. Now on the mobile phone market, many brands are using the Android mobile phone system, launched their own Android mobile phones, China mobile and China Unicom 
and other operators are developed based on the Android mobile operating platform. The Android system in people daily life has been occupies an important position. Android application is composed of loose components of affinity, and using the application list together, the application list describes each component, the interactive relationship between them, as well as the application metadata. The Android operating system has the complete API and open nature, as shown in figure 1.

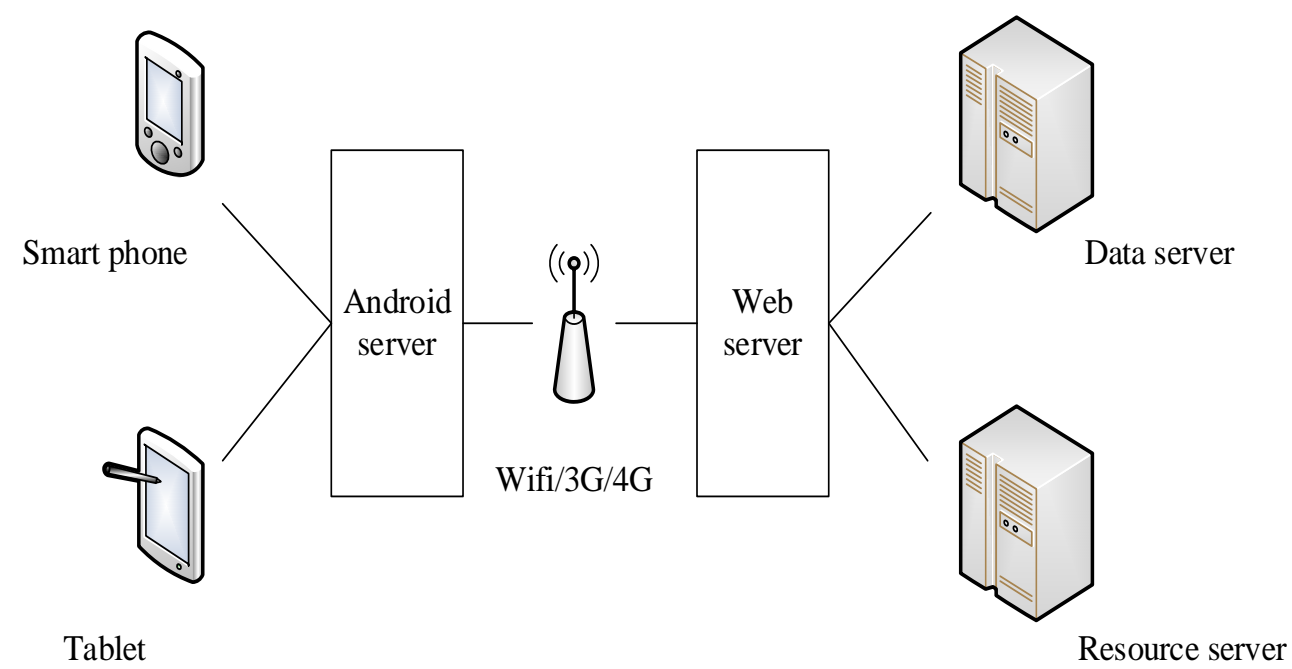

Figure 1. Mobile learning system framework based on the Android platform

Mobile learning system customers include portable smart device Android mobile phone and Android tablet computer, users' access to their learning and other learning courses and related operation through mobile devices. Mobile learning system server mainly consists of two parts, the database server and server resources [5]. The main function of the database server is to store related to students' personal information, legitimacy and is responsible for the correctness verification and feedback of the users to submit personal information. Thus the curriculum resources (super text, pictures, animation, streaming media etc.) and processing of online interactive information, including the student asked the teacher to answer questions, etc.

\section{Analysis of mobile teaching platform based on the smart phone}

As this system based on Android mobile learning platform of experimental research, its main goal is to design and develop a simple and reliable mobile learning platform, in the process of the design of the system mainly follows the expansibility principle, feasibility principle, the principle of practicability and security. The design of this system is to make full use of the existing curriculum resources, under the premise that ensure the safety of communications, design and let the hair with a real-time, fast and easy to maintain, expand and update the mobile learning platform, so that students and teachers can at any time, any place for themselves to the knowledge of information, realize in the true sense of autonomous learning. Mobile learning platform need to pass the requirements analysis, design and realization of the platform design and platform to realize the three stages, which platform is the design and implementation of two parallel stage, its flow chart is shown in figure 2.

The design of the mobile learning platform mainly including server and client design. Server-side design mainly includes the overall design of architecture design, architecture design and function design, base class design, module design; The client's design including functional design, UI design, base class design, module design. The design on the base of this chapter on the server class design and module design, in the client's design focuses on the function design and UI design. 


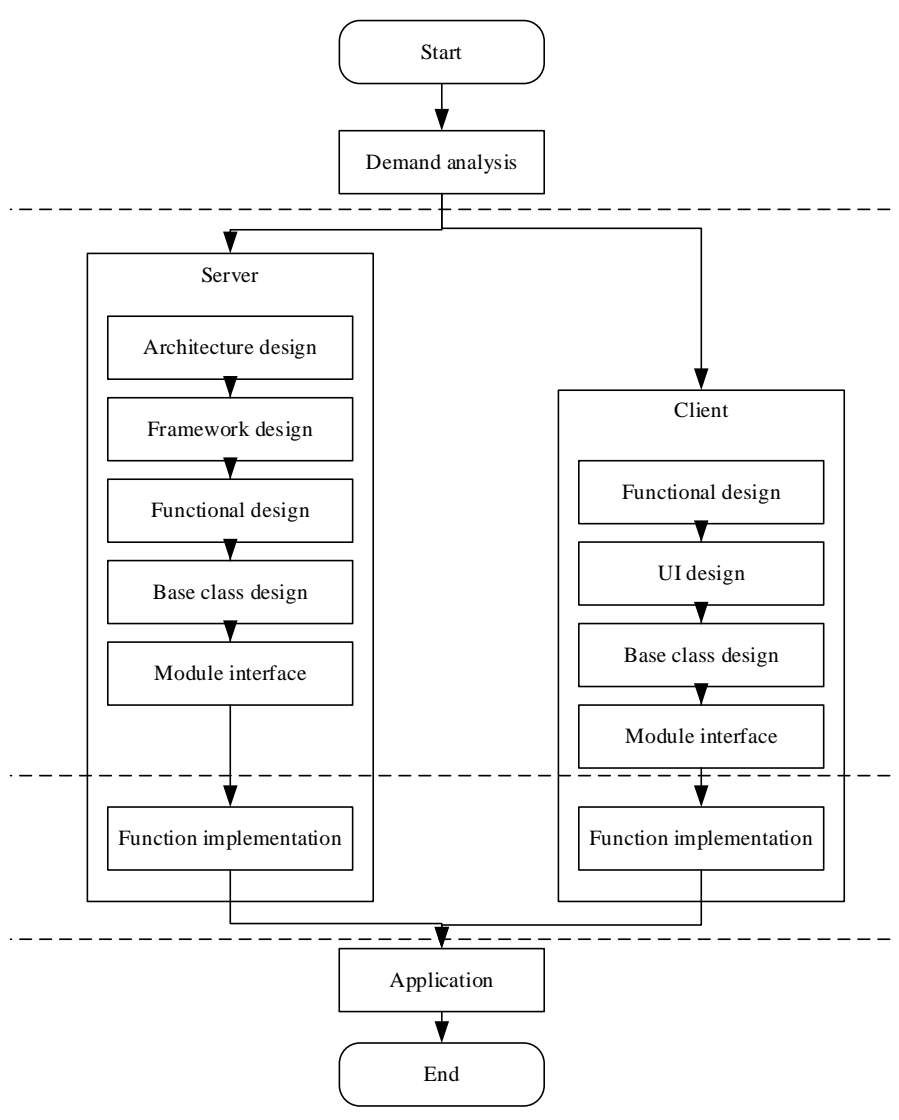

Figure 2. The design process of mobile teaching platform based on the smart phone

\section{Hardware architecture of mobile teaching platform based on the smart phone}

With the rapid development of mobile Internet and the growing popularity of the intelligent mobile terminal equipment, for the development on the intelligent mobile terminal software provided unprecedented market space. Teaching methods can no longer be confined to the traditional mode, teachers should enrich the teaching tools, make full use of high-tech to provide us with the network environment and communication equipment, improve teaching efficiency. Mobile teaching platform based on smart phones using C/S (Client/Server) mode is the development of targeted, interactive, with safe access module, fast response speed, the Client application to install and use simple operation. Mobile teaching platform based on smart phones are divided into two subsystems side and the teachers and students, the system design on the same structure, on the specific module implements is different. Mobile teaching platform based on smart phone hardware structure diagram is shown in figure 3 .

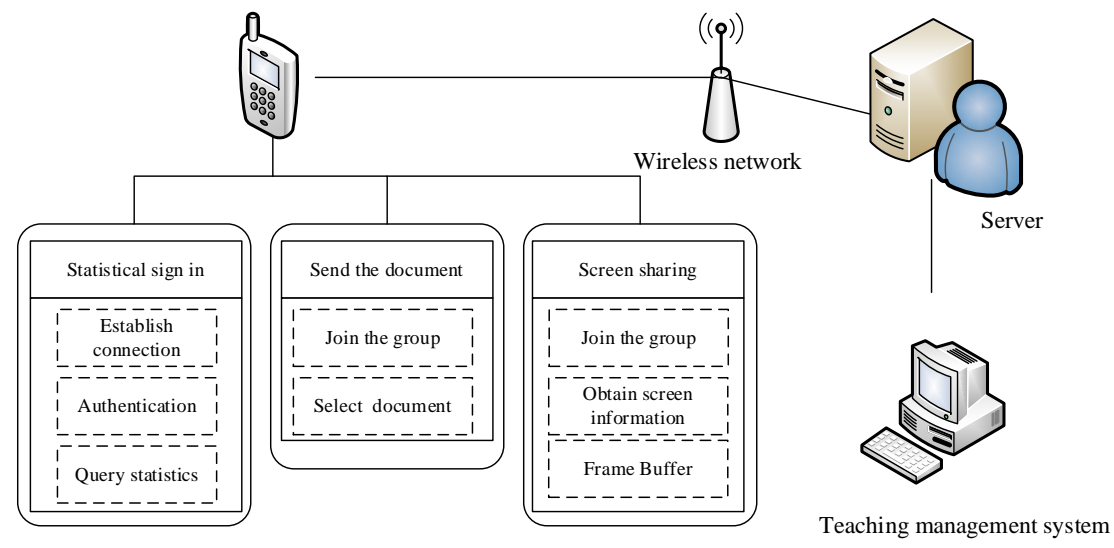

Figure 3. Hardware architecture of mobile teaching platform based on the smart phone 
Student's terminal system server provides the students check-in service data sources and processing operations, students complete check-in by connecting to the server. Design and development of the part is run on the Android students sign in three program, receiving documents, screen sharing. The three programs as a student terminal system with the server client access to the wireless network to obtain IP address connection is established, through the corresponding query algorithm for students of today's schedule information, select courses, encapsulated in the data format to check in. File transfer, first of all, based on Android platform from the server side to obtain corresponding courses according to teacher's ID assigned IP multicast group, to join the multicast group, after decoding and then receiving electronic documents. In the form of document flow implementation screen sharing need to gain root access, by manipulating the device files to complete the update of the screen. Also need to consider the software using the cell phone memory card capacity in the process of problem, so, in the software design phase using regular update of the screen method did not take the traditional after frame using the human eye persistence of vision in the form of pictures sent to the client, the client again in the form of a slide show, this approach requires the client program regularly delete the old pictures.

\section{Conclusion}

With the rapid development of mobile computing technology, a new distance learning mode arises at the historic moment, this is the mobile learning. As a new research field, mobile learning on the basis of digital learning through effective combination of mobile computing technology, make the learners can at any time any place you want to get knowledge, to realize in the true sense of autonomous learning, finally realizes the socialized and lifelong learning. In this paper, based on the research of intelligent mobile phone mobile teaching platform, which is based on mobile learning theory, analysis of the mobile user needs of learners, the theory of mobile learning platform and software model is set up, and then application based on Android mobile platform development technology, design and development of mobile learning platform for mobile learning platform design research to provide certain reference, and for the mobile learning platform to provide reference for the construction of let hair and so it has important research value and broad application prospects.

\section{References}

[1] J. Sandberg, M. Maris, K. Geus: Computers \& Education, Vol.57 (2011) No.1, p. 1334.

[2] R. Godwin-Jones: Language Learning \& Technology, Vol.15 (2011) No.2, p. 11.

[3] L. Hsu: Computer Assisted Language Learning, Vol.26 (2013) No.3, p. 197.

[4] A. Kukulska-Hulme: Journal of Educational Technology and Society, Vol.13 (2010) No.4, p. 4.

[5] Y. Tai: Educational Technology \& Society, Vol.15 (2012) No.2, p. 220. 